\title{
Granzyme $M$ has a critical role in providing innate immune protection in ulcerative colitis
}

\author{
F Souza-Fonseca-Guimaraes ${ }^{*, 1,2,3}$, Y Krasnova ${ }^{1,4}$, T Putoczki ${ }^{5}$, K Miles ${ }^{1}$, KP MacDonald ${ }^{6}$, L Town ${ }^{1}$, W Shi ${ }^{7}$, GC Gobe ${ }^{8}$, L McDade ${ }^{1}$, \\ LA Mielke ${ }^{3}$, H Tye ${ }^{5}$, SL Masters ${ }^{5}$, GT Belz ${ }^{3}$, ND Huntington ${ }^{3}$, G Radford-Smith ${ }^{9,10,11}$ and MJ Smyth ${ }^{\star, 1,4,11}$
}

Inflammatory bowel disease (IBD) is an immunoregulatory disorder, associated with a chronic and inappropriate mucosal immune response to commensal bacteria, underlying disease states such as ulcerative colitis (UC) and Crohn's disease (CD) in humans. Granzyme M (GrzM) is a serine protease expressed by cytotoxic lymphocytes, in particular natural killer (NK) cells. Granzymes are thought to be involved in triggering cell death in eukaryotic target cells; however, some evidence supports their role in inflammation. The role of GrzM in the innate immune response to mucosal inflammation has never been examined. Here, we discover that patients with UC, unlike patients with CD, display high levels of GrzM mRNA expression in the inflamed colon. By taking advantage of well-established models of experimental UC, we revealed that GrzM-deficient mice have greater levels of inflammatory indicators during dextran sulfate sodium (DSS)-induced IBD, including increased weight loss, greater colon length reduction and more severe intestinal histopathology. The absence of GrzM expression also had effects on gut permeability, tissue cytokine/chemokine dynamics, and neutrophil infiltration during disease. These findings demonstrate, for the first time, that GrzM has a critical role during early stages of inflammation in UC, and that in its absence colonic inflammation is enhanced.

Cell Death and Disease (2016) 7, e2302; doi:10.1038/cddis.2016.215; published online 21 July 2016

Inflammatory bowel disease (IBD) is a gut-associated inflammatory disorder, which stems from a dysfunctional mucosal immune response to commensal bacteria. ${ }^{1}$ As a multifactorial disease, IBD is the consequence of a complex interplay between environmental triggers, genetic susceptibility, and immunoregulatory defects, resulting in a pathogenesis that is still poorly understood. ${ }^{2}$ These interactions result in the inability of an individual to control the normal inflammatory response to pathogens in the gut, leading to a chronic state of sustained and inappropriate inflammation. IBD underlies disease states such as ulcerative colitis (UC) and Crohn's disease (CD), with symptoms including weight loss, abdominal pain, diarrhea, and rectal bleeding which often require intensive medical therapy and resective surgery. ${ }^{3}$ The pathogenesis of IBD, characterized by a defective mucosal immune response to microbial exposure in the gastrointestinal tract, is thought to be caused by a dysfunctional immune response to host microbiota, infection by specific pathogens, and/or a defective mucosal barrier to luminal pathogens. ${ }^{1,2}$ IBD patients also have a high risk of developing colitisassociated colon cancer (CAC). ${ }^{4}$ Additionally, histological assessment of inflamed ileal and colonic segments from IBD patients typically shows increased infiltration of immune cells, particularly neutrophils, as well as crypt abscesses, mucin depletion, and ulcers - all correlating with the severity of small bowel and colonic tissue damage. ${ }^{5}$

Cytotoxic pathways mediated by lymphocytes directly trigger cell death in target cells. ${ }^{6}$ These cytotoxic pathways are mediated by proteins such as perforin, which mediates pore formation in the target cell surface and allows granzyme (Grz)s to enter the intracellular compartment and induce cell death. ${ }^{7}$ To date, five different Grzs have been identified in humans (GrzA, GrzB, GrzH, GrzK, and GrzM), whereas mice express eleven Grzs (GrzA, GrzB, GrzC, GrzD, GrzE, GrzF, GrzG, GrzK, GrzL, GrzM, and GrzN). ${ }^{8,9}$ Walch et al. ${ }^{10}$ recently demonstrated that Grzs (GrzA and GrzB) directly kill bacteria through granulysin-mediated delivery, suggesting that Grzs act as microbial modulating factors. Moreover, recently GrzA was shown to be increased in the colon biopsies of UC patients undergoing treatment with Etrolizumab, a monoclonal antibody targeting the $\beta 7$ integrin subunit. Higher levels of GrzA could predict which patients were more likely to benefit

\footnotetext{
${ }^{1}$ Immunology in Cancer and Infection Laboratory, QIMR Berghofer Medical Research Institute, Herston, Queensland 4006, Australia; ${ }^{2}$ Faculty of Medicine, Dentistry and Health Sciences, University of Melbourne, Melbourne, Victoria 3010, Australia; ${ }^{3}$ Division of Molecular Immunology, The Walter and Eliza Hall Institute of Medical Research, Parkville, Victoria 3052, Australia; ${ }^{4}$ School of Medicine, University of Queensland, St Lucia, Queensland 4006, Australia; ${ }^{5}$ Inflammation Division, The Walter and Eliza Hall Institute of Medical Research, Parkville, Victoria 3052, Australia; ${ }^{6}$ Antigen Presentation and Immunoregulation Laboratory, QIMR Berghofer Medical Research Institute, Herston, Queensland 4006, Australia; ${ }^{7}$ Signal Transduction Laboratory, QIMR Berghofer Medical Research Institute, Herston, Queensland 4006, Australia; ${ }^{8}$ Centre for Kidney Disease Research, School of Medicine, University of Queensland at Translational Research Institute, St Lucia, Queensland 4006, Australia; ${ }^{9}$ Inflammatory Bowel Disease Laboratory, QIMR Berghofer Medical Research Institute, Herston, Queensland 4006, Australia and ${ }^{10}$ Department of Gastroenterology, Royal Brisbane and Women's Hospital, Herston, Queensland 4006, Australia

${ }^{*}$ Corresponding author: F Souza-Fonseca-Guimaraes or MJ Smyth, Immunology in Cancer and Infection Laboratory, QIMR Berghofer Medical Research Institute, 300 Herston Road, Herston, Queensland 4006, Australia. Tel: +61 73845 3957; Fax: +61 7 38453957; E-mail: guimaraes.f@wehi.edu.au or Mark.Smyth@ qimrberghofer.edu.au ${ }^{11}$ These authors contributed equally to this work.

Abbreviations: AOM, azoxymethane; CD, Crohn's disease; CAC, colitis-associated colon cancer; CRC, colorectal cancer; DSS, dextran sulfate sodium; EMT, epithelialmesenchymal transition; Grz, granzyme; IBD, inflammatory bowel disease; IEL, intraepithelial leukocyte; ILC, innate lymphoid cell; LP, lamina propria; NK, natural killer; Prf1, perforin; RBC, red blood cell; UC, ulcerative colitis; WT, wild type Received 15.2.16; revised 03.6.16; accepted 06.6.16; Edited by T Brunner
} 
from the therapy; however, the precise mechanism of action of GrzA in UC remains to be addressed. ${ }^{11}$ GrzM was initially described as being constitutively expressed by natural killer (NK) cells, $^{12,13}$ and specifically associated with inflammation. ${ }^{14}$ This enzyme has been shown to preferentially cleave methionine and leucine residues in target cells, mediating direct, non-specific cell death. ${ }^{15,16}$ More recently, GrzM was also shown to be an important mediator for the release of MIP-1a from NK cells, inducing NK cell and neutrophil recruitment during early microbial infection. ${ }^{17} \mathrm{We}$ now observe that GrzM expression is increased in inflamed colon tissue samples from UC, but not CD patients. Further, GrzM-deficient $\left(\mathrm{GrzM}^{-/-}\right)$mice are more sensitive to a mouse model of IBD and IBD-induced colorectal cancer (CRC). These findings demonstrate, for the first time, that GrzM has a critical role in mediating the early stages of the gut mucosal immune response.

\section{Results}

GrzM expression is increased in the inflamed rectal tissues from UC patients. mRNA analysis of mucosal tissue biopsies from UC patients has been identified as a potential tool to investigate gene expression at different time points in this disease. ${ }^{11}$ To investigate whether GrzM may either be present or highly expressed in the inflamed intestinal segments of IBD patients, biopsies from inflamed and non-inflamed regions of the cecum, transverse colon, sigmoid colon, and rectum of UC patients; and from inflamed and non-inflamed regions of the ileum of CD patients were compared with tissue biopsies matched for intestinal segments from healthy controls. Biopsies were obtained at the time of colonoscopy, processed for tissue mRNA extraction and analyzed for GrzM mRNA (Table 1). Notably, GrzM expression was elevated specifically in the inflamed tissues of the rectal portions of UC patients, while no difference was detected in CD patients.

GrzM is critical for colonic integrity during experimental dextran sulfate sodium-induced colitis. Following on from our finding that GrzM expression was elevated in the rectal tissues of UC patients, we set out to investigate whether GrzM expression was favorable or unfavorable in the UC disease course. By taking advantage of the GrzM-deficient mice previously described by our group, ${ }^{18}$ we performed a screen with dextran sulfate sodium (DSS)-induced colitis (Figure 1a), a mouse experimental model of human UC. ${ }^{19}$ We observed that $\mathrm{GrzM}^{-/-}$mice were susceptible to weight loss following a $5 \%$ DSS dose (Figure $1 \mathrm{~b}$ ). In concert, by using a well-established histopathology characterization and scoring system, ${ }^{20}$ we observed that the central-distal portion of the colon of $\mathrm{GrzM}^{-1-}$ mice post DSS treatment was dramatically affected (Figures 1c and d). In agreement with the increased gut pathology, enhanced neutrophil recruitment was detected in the lamina propria region post DSS treatment (Figure 1e). To investigate whether the enhanced colonic disease phenotype seen in GrzM-deficient mice would also be observed in other experimental models that are dependent on gut integrity, we performed experiments using Toxoplasma gondi ${ }^{21}$ and Citrobacter rodendium; ${ }^{22}$ however, we failed to detect any difference between WT and $\mathrm{GrzM}^{-1-}$ mice in these conditions (data not shown).

\section{GrzM expression inhibits excessive neutrophil infiltration} and promotes gut integrity. Neutrophils are critical for bacterial leakage control and maintenance of homeostasis; however, excessive recruitment and activation of these cells may trigger mucosal injury and consequently worsen disease symptoms. $^{23}$ To verify whether GrzM deletion increased the colonic infiltration by neutrophils at the steady state or during DSS-induced colitis, homogenates of the intraepithelial portion of the colon and lamina propria were assessed. In concert, GrzM-deficient mice displayed increased neutrophil infiltration, especially in lamina propria fractions after DSS challenge (Figures $2 \mathrm{a}-\mathrm{c}$ ). As a consequence of excessive neutrophil activation, gut integrity can be damaged during IBD, and can allow luminal antigen and bacterial leakage into the subepithelial tissues, resulting in enhanced inflammation. ${ }^{24}$ To investigate whether gut integrity is affected in the steady state or whether the damage is increased following DSS treatment, we assessed intestinal permeability using an FITC-dextran absorption assay. ${ }^{25}$ Gut integrity was preserved in $\mathrm{GrzM}^{-1-}$ mice during the steady state; however, a dramatically elevated translocation of FITC-dextran from gut to the circulation was detected when these mice were challenged with DSS, suggesting that the presence of GrzM is critical for gut integrity (Figure 2d). The gut integrity dysfunction followed by induction of inflammation triggers increased neutrophil chemotaxis and infiltration into the inflamed tissue.

In addition, to investigate whether colonic lamina propria or intraepithelial cells, specifically in the proximal or distal fractions, were also infiltrated by lymphocyte subsets (previously described as potential expressers of $\mathrm{GrzM}^{26}$ ), we also assessed the infiltration of CD8 T cells, $\gamma \delta$ T cells, or Group 1 innate lymphoid cells (which incorporate NK cells and ILC1 ${ }^{27}$ ) post DSS in WT mice. The gating strategy for the quantification of these cell subsets from colonic tissue is shown in Supplementary Figure 1. In agreement with the increased neutrophil infiltration, all these lymphocyte subsets were increased in numbers in the lamina propria in both distal and proximal parts (Figure $2 \mathrm{e}$ ). In the intraepithelial fraction, predominant CD8 T cells and NK cells were increased in the intraepithelial fractions in both distal and proximal parts, while $\gamma \delta \mathrm{T}$ cells were in higher levels in the proximal part and ILC1s increased in the distal part. Although all the potential GrzMexpressing lymphocyte subsets were increased in the colonic tissue post DSS challenge, the specific deficiency or depletion of CD8 T cells, $\gamma \delta$ T cells, or NK/ILC1 cells did not mimic the GrzM-deficient mice phenotype (Supplementary Figure 2AD). Perforin (Prf1) is a molecule expressed by cytotoxic lymphocytes that are used in killing by transiently inducing pore formation target cells and allowing Grzs to be internalized and induce cell death. ${ }^{28}$ Although perforin can act with Grzs to induce cytotoxic events, the effects of GrzM in UC appeared to be perforin independent, since the Prf1-deficient mice displayed no enhancement of colitis post DSS challenge (Supplementary Figure 2E). Colon length is a quantitative measure of disease severity where the extent of inflammation 
Role of GrzM in innate immune protection in UC

Table 1 High levels of GrzM expression found exclusively in inflamed rectum portions of UC patients

\begin{tabular}{|c|c|c|c|c|c|c|c|c|}
\hline & \multirow[b]{2}{*}{$N$} & \multirow[b]{2}{*}{ Mean } & \multirow[b]{2}{*}{ S.D. } & \multirow[b]{2}{*}{ S.E. } & \multirow[b]{2}{*}{ Lower bound } & \multirow[b]{2}{*}{ Upper bound } & \multicolumn{2}{|c|}{$95 \% \mathrm{Cl}$} \\
\hline & & & & & & & $P$-value & P.adjust \\
\hline \multicolumn{9}{|c|}{ Transverse colon in UC } \\
\hline C & 22 & 6.47 & 0.40 & 0.09 & 6.29 & 6.65 & & \\
\hline UC.I & 15 & 6.43 & 0.30 & 0.08 & 6.27 & 6.60 & 0.048 & 0.338 \\
\hline UC.NI & 17 & 6.21 & 0.25 & 0.06 & 6.08 & 6.34 & & \\
\hline Total & 54 & 6.38 & 0.35 & 0.05 & 6.28 & 6.47 & & \\
\hline \multicolumn{9}{|c|}{ Sigmoid in UC } \\
\hline C & 22 & 6.32 & 0.29 & 0.06 & 6.19 & 6.45 & & \\
\hline UC.I & 32 & 6.42 & 0.32 & 0.06 & 6.31 & 6.53 & 0.335 & 0.361 \\
\hline UC.NI & 14 & 6.31 & 0.21 & 0.06 & 6.19 & 6.43 & & \\
\hline Total & 68 & 6.36 & 0.29 & 0.03 & 6.29 & 6.43 & & \\
\hline \multicolumn{9}{|c|}{ Rectum in UC } \\
\hline & 22 & 6.29 & 0.18 & 0.04 & 6.21 & 6.37 & & \\
\hline UC.I & 29 & 6.51 & 0.41 & 0.08 & 6.35 & 6.67 & $0.018^{*}$ & $0.028^{*}$ \\
\hline UC.NI & 11 & 6.26 & 0.13 & 0.04 & 6.18 & 6.35 & & \\
\hline Total & 62 & 6.39 & 0.32 & 0.04 & 6.31 & 6.47 & & \\
\hline \multicolumn{9}{|c|}{ Cecum in UC } \\
\hline C & 22 & 6.38 & 0.29 & 0.06 & 6.25 & 6.50 & & \\
\hline UC.I & 10 & 6.28 & 0.16 & 0.05 & 6.16 & 6.39 & 0.201 & 0.403 \\
\hline UC.NI & 16 & 6.25 & 0.13 & 0.03 & 6.18 & 6.32 & & \\
\hline Total & 48 & 6.31 & 0.23 & 0.03 & 6.25 & 6.38 & & \\
\hline \multicolumn{9}{|c|}{ Ileum in CD (non-inflamed) } \\
\hline C & 21 & 6.609 & 0.424 & 0.093 & 6.416 & 6.802 & & \\
\hline CD.NI & 21 & 6.572 & 0.386 & 0.084 & 6.396 & 6.747 & 0.766 & 0.825 \\
\hline Total & 42 & 6.590 & 0.401 & 0.062 & 6.465 & 6.715 & & \\
\hline \multicolumn{9}{|c|}{ Ileum in CD (inflamed) } \\
\hline & 21 & 6.609 & 0.424 & 0.093 & 6.416 & 6.802 & & \\
\hline CD.I & 25 & 6.429 & 0.390 & 0.078 & 6.268 & 6.590 & 0.140 & 0.656 \\
\hline Total & 46 & 6.511 & 0.411 & 0.061 & 6.389 & 6.633 & & \\
\hline
\end{tabular}

Samples from inflamed (I) or non-inflamed (NI) areas from different anatomical parts of the colons of ulcerative colitis (UC: transverse, sigmoid, rectum, and cecum) or Crohn's disease (CD: lleum) patients were screened and analyzed for GrzM mRNA expression. Statistical analysis was performed using one-way ANOVA, where ${ }^{\star} P<0.05$ was considered for statistical significance.

directly correlates to increased edema, leading to an overall shortening of the colon. $^{29}$ In concert, elevation of proinflammatory cytokines also correlates with increased disease pathology in IBD. ${ }^{30}$ Although we observed enhanced colon length shrinkage in GrzM- and TCR $\delta$-deficient mice after DSSinduced UC, in agreement with the weight loss data we only observed elevation of G-CSF and TNF in the serum of GrzMdeficient mice (Supplementary Figure 2F-H). Our results indicate that the GrzM-mediated colonic protection involves a mechanism that might require a complex participation of multiple factors to promote protection in UC.

GrzM expression protects colonic epithelium from inflammation-induced CRC. We next assessed whether $\mathrm{GrzM}^{-1-}$ mice would also be more susceptible to colon cancer development. Colonic epithelial oncogenesis can either be developed by sporadic progression due to genetic predisposition or following an inflammation-associated trigger. ${ }^{31}$ Azoxymethane $(\mathrm{AOM})$ is a highly mutagenic chemical compound that causes K-ras mutations. When used repeatedly via intraperitoneal administration, it can trigger sporadic CRC, and when used before DSS-induced inflammation it can trigger inflammation-associated CRC. ${ }^{32}$ We failed to observe any phenotype of sporadic CRC predisposition in $\mathrm{GrzM}^{-1-}$ mice in response to repetitive $\mathrm{AOM}$ treatment (data not shown).
However, when the inflammation-induced CRC model was examined (Figure 3a), the $\mathrm{GrzM}^{-/-}$mice clearly displayed susceptibility to the AOM-DSS-induced CRC demonstrated by enhanced weight loss cycles (Figure 3b). As expected, GrzMdeficient mice also displayed an increased number of macroscopically visible colorectal polyps (Figures $3 c$ and d). Colon length was another indicator of increased inflammation, since we observed a significant shortening of colon length in GrzM-deficient mice compared with the wild-type (WT) control mice at the same stages of inflammation (Figure 3e). This suggests that the severity of inflammation during the AOMDSS model was enhanced in GrzM-deficient mice. This was corroborated by the measurement of an enhanced histopathology scoring of the middle parts of both mucosa and submucosa sections in the colon of GrzM-deficient mice at the end point of the experiment (Figure 3f). We also observed that the effects of AOM-DSS in GrzM-deficient mice had a systemic impact, since the mice displayed increased mesenteric lymph nodes and spleen sizes, as well as increased leukocyte numbers in both compartments (data not shown).

Regarding the potential GrzM expression by colonic epithelial cells, Wang et al. ${ }^{33}$ recently showed mouse GrzM detection in mouse colon cancer cells using the anti-mouse GrzM antibody clone aa31-257. In addition to this, another anti-mouse GrzM antibody clone P-15 also recently became 


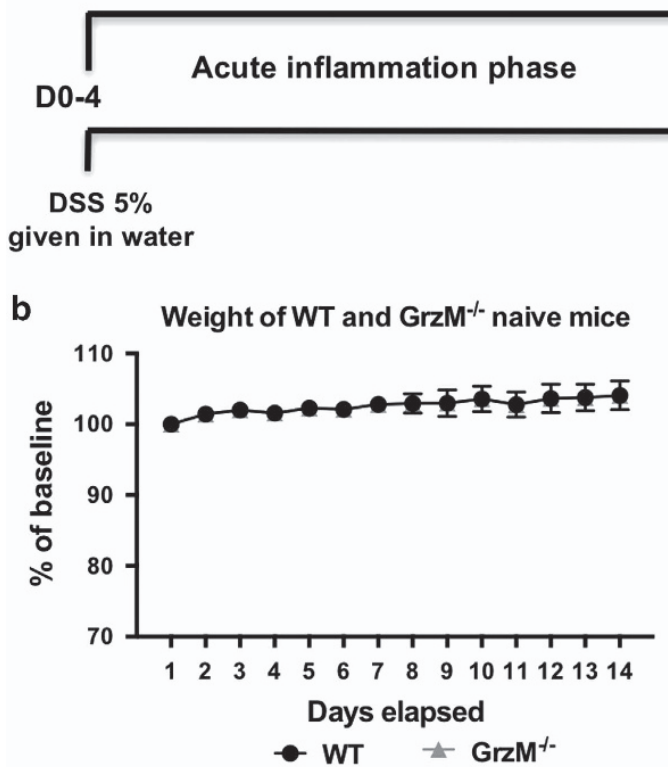

D7

$\gamma_{10}$

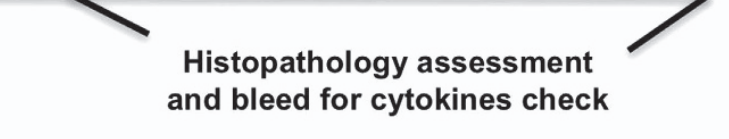

Histopathology assessment and bleed for cytokines check

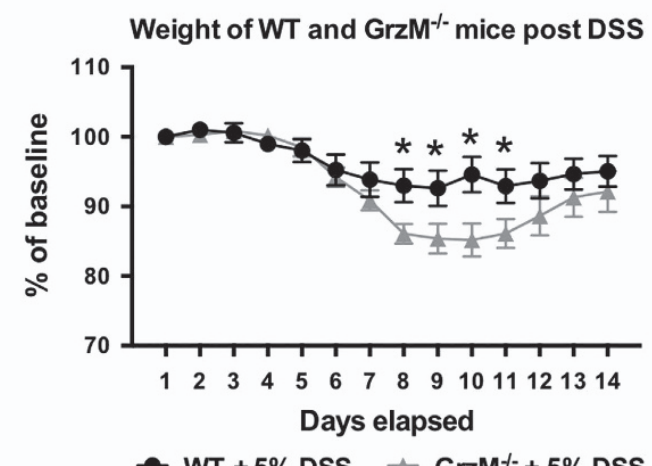

C

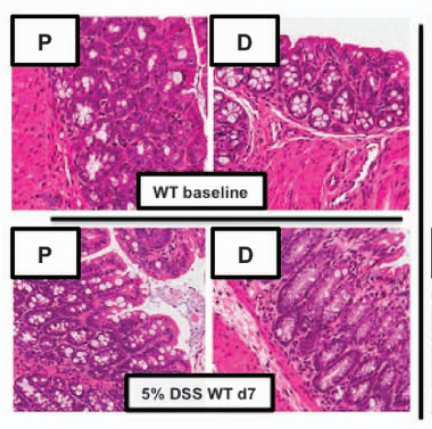

e

d7-WT

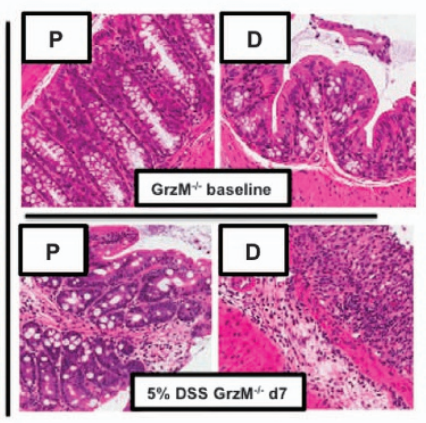

d
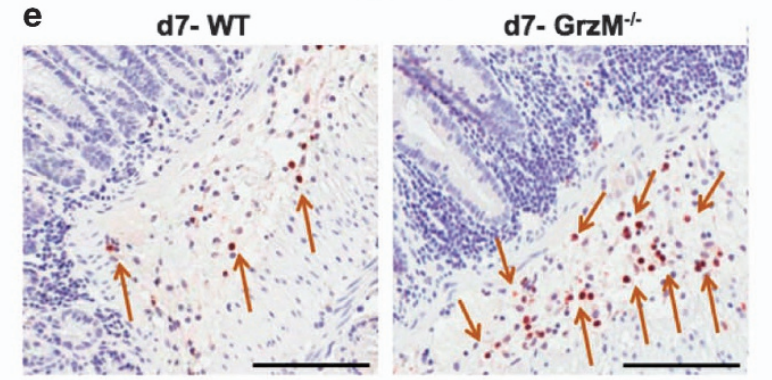

\section{Proximal}
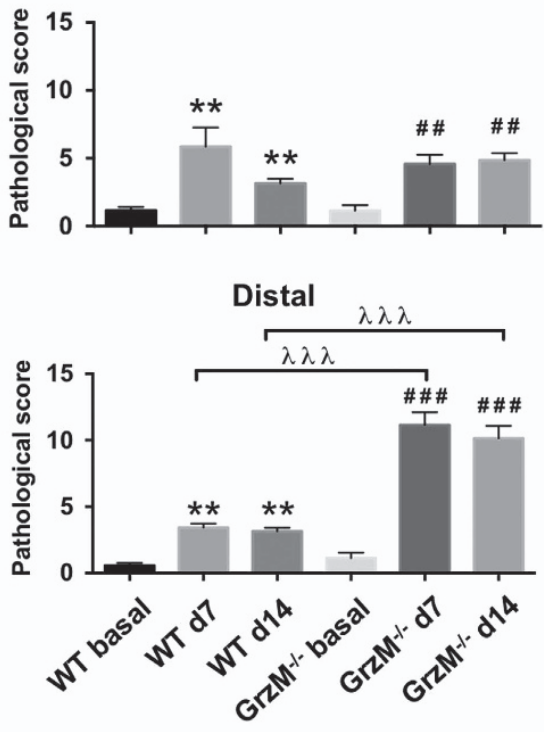

Figure 1 DSS-induced colitis reveals GrzM as a critical factor for disease protection. Mice were challenged with $5 \%$ DSS in the drinking water for 4 days, and samples were assessed 7 or 14 days post first DSS treatment day, according to the experimental design proposed in (a). (b) DSS-induced weight loss was measured daily throughout the 14-day experimental period. Statistical analysis was performed using Mann-Whitney test. Results are representative from pool of two experiments, and are expressed in mean \pm S.D. $n=5$ mice per experiment ( $n=10$ total), and ${ }^{*} P<0.05$ was considered for statistical significance. (c) Representative histological sections (HE stain) from the proximal (P) and distal (D) colon parts from WT or GrzM-deficient mice at baseline, 7 days post $5 \%$ DSS (d7), and 14 days post 5\% DSS (d14). (d) Histopathological scoring quantification from colons post $5 \%$ DSS. Statistical analysis was performed using Mann-Whitney test. Results are expressed in mean \pm S.E.M. $n=6$ mice per group, ${ }^{\star} P<0.05$, ${ }^{* \star}$ (or \#\#) $P<0.01$, and ${ }^{* * *}$ (or \#\#\# or $\left.\lambda \lambda \lambda\right) P<0.001$. ${ }^{*}$ Comparison between WT group, " comparison between GrzM-deficient group, and ${ }^{\lambda}$ comparison between WT and GrzM-deficient groups. (e) Ly6G immunohistochemistry illustrates the increased neutrophil (Ly6G+ ${ }^{+}$revealed by DAB stain (brown die, indicated by brown arrows) in representative areas of the colonic lamina propria of WT and GrzM-deficient mice post 7 days after DSS challenge

commercially available. To investigate whether these available tools could help us decipher in which exact colon cellular compartment GrzM is expressed, we initially assessed whether the staining of both aa31-257 and P-15 antibody clones was specific to mouse GrzM. We performed tissue protein extraction from the colons of WT and GrzM-deficient mice and subsequently performed western blot detection according to the manufacturer's instructions. However, we found that both aa31-257 and P-15 clones were highly nonspecific, as there was staining in all the $\mathrm{GrzM}^{-/-}$samples 
a

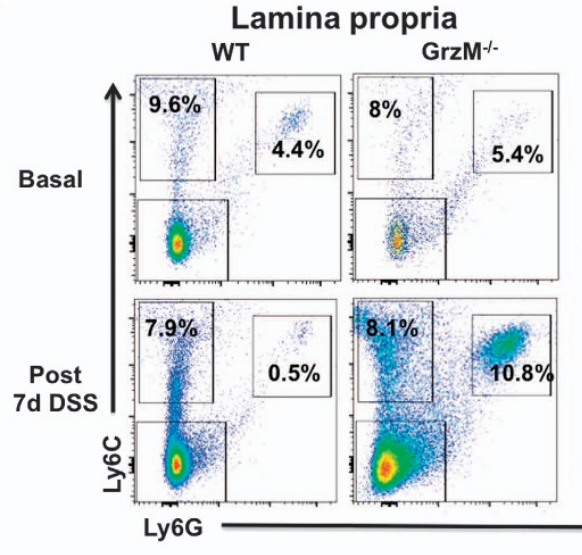

IEL / LP neutrophil (Ly6G+/Ly6C + ) comparison

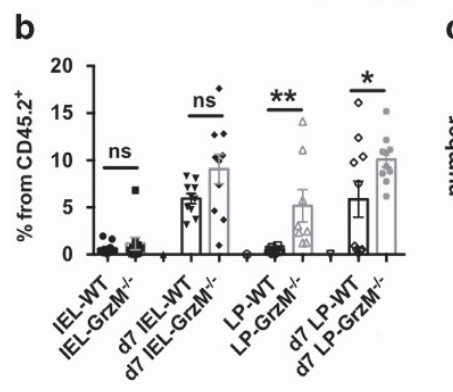

Intra-epithelial WT GrzM-

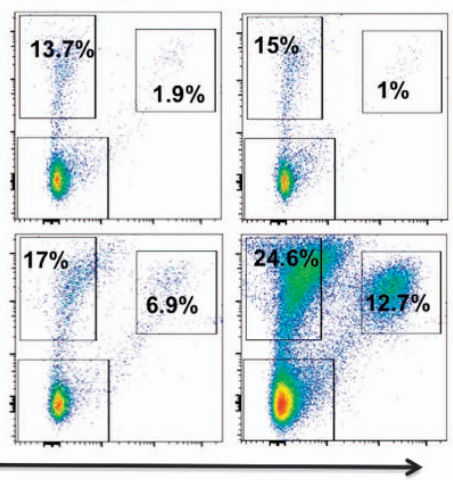

C

e

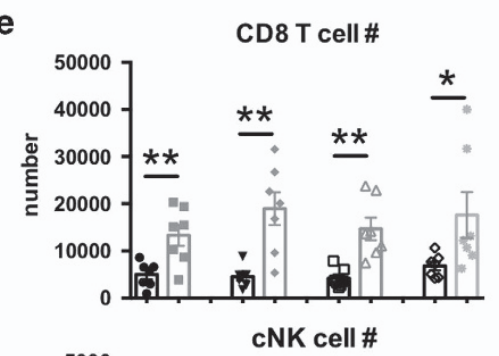

b

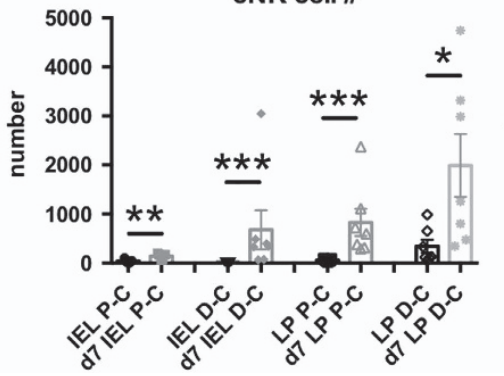

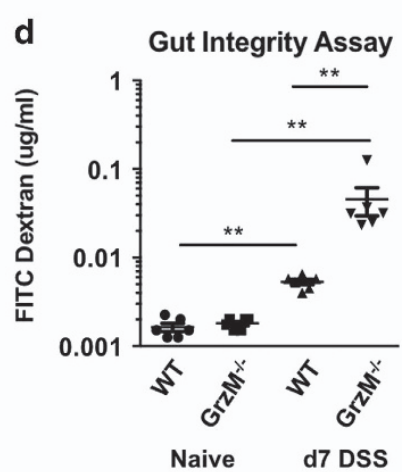
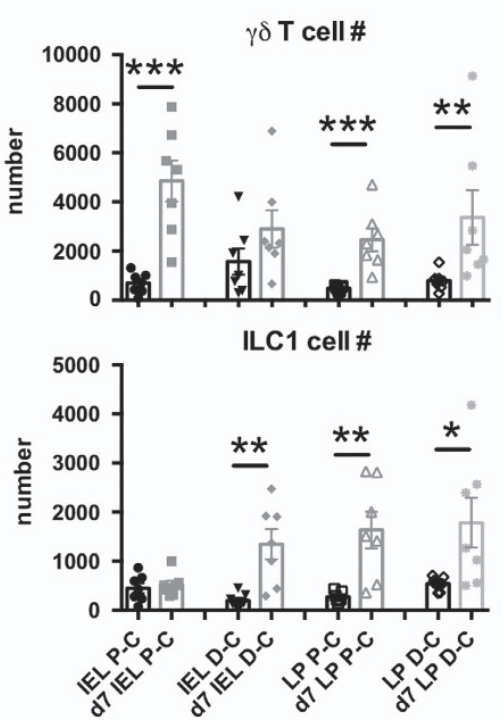

Figure 2 DSS treatment induced higher neutrophil infiltration in colons of GrzM-deficient mice. (a) Representative dot blots from both colon lamina propria and intraepithelial phase of WTand GrzM-deficient mice pre and post 7 days of $(7 \mathrm{~d}) 5 \%$ DSS treatment. Neutrophils were gated from leukocyte gate $\left(\mathrm{CD} 45.2^{+}\right)$as Ly6C ${ }^{+} \mathrm{Ly} 6 \mathrm{G}^{+}$. (b) Neutrophils were compared within their respective \% of CD45.2+ leukocytes from both lamina propria (LP) and intraepithelial (IEL) parts. (c) Bead-based absolute counting was utilized to quantify neutrophils from both lamina propria and epithelium. Results are expressed in mean \pm S.E.M., and ${ }^{*} P<0.05$, ${ }^{* *} P<0.01$, and ${ }^{* * *} P<0.001$ were considered as statistically significant by Mann-Whitney test. Results are representative from the pool of two independent experiments with $n=5$ in each group ( $n=10$, total). (d) Gut integrity of naive WT and GrzM-deficient animals, or post 7 days after DSS challenge, was assessed by measuring the in vivo gut permeability post oral gavage administration of FITC-dextran for $4 \mathrm{~h}$ and then respective FITC detection in plasma. Results are expressed in mean \pm S.E.M., and ${ }^{\star \star} P<0.01$ was considered as statistically significant by Mann-Whitney test. Results are representative from the pool of two experiments with $n=3$ in each group ( $n=6$, total). (e) CD8 T cell, $\gamma \delta$ T cell, conventional NK (cNK), and ILC1 cell quantification from the proximal-central (PC) and distal-central (DC) parts of IEL and LP colonic fractions, post 7 days after DSS challenge in WT mice. Results are expressed in mean \pm S.E.M., and ${ }^{*} P<0.05$, ${ }^{\star \star} P<0.01$, or ${ }^{\star \star *} P<0.001$ was considered as statistically significant by Mann-Whitney test. Results are representative from $n=7$ independent biological replicates for each group 


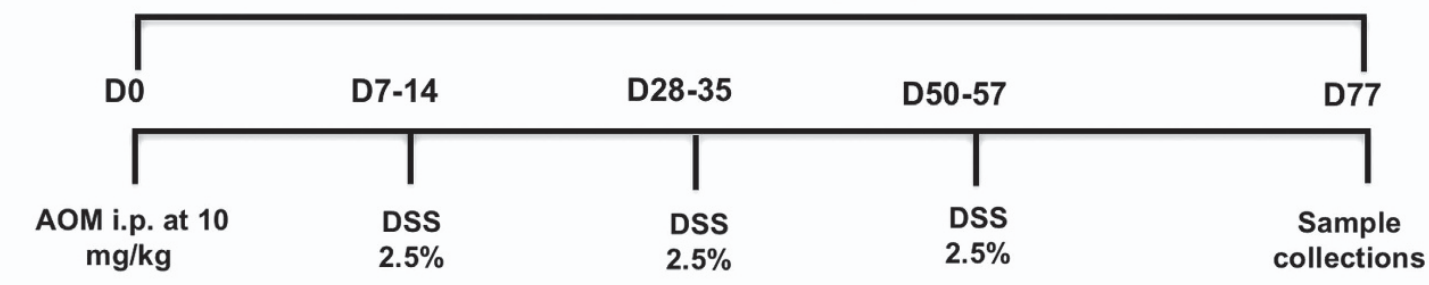

b

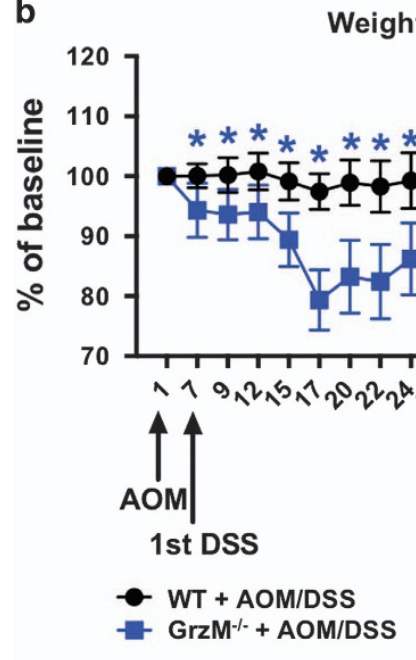

d

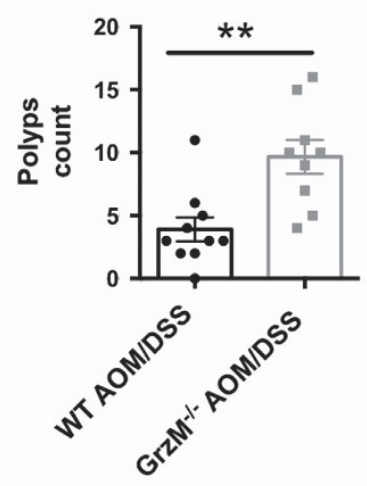

Weight post AOM/DSS treatment

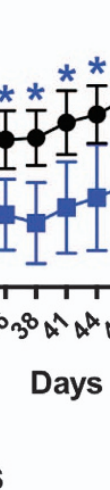

2nd DSS

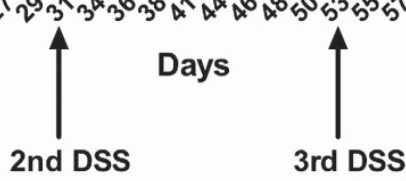

e

Colon length
post AOM/DSS

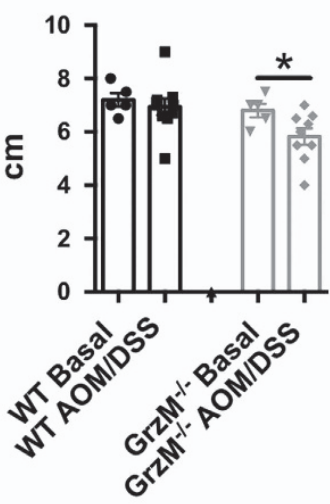

f

Colon histopathological score post AOM/DSS

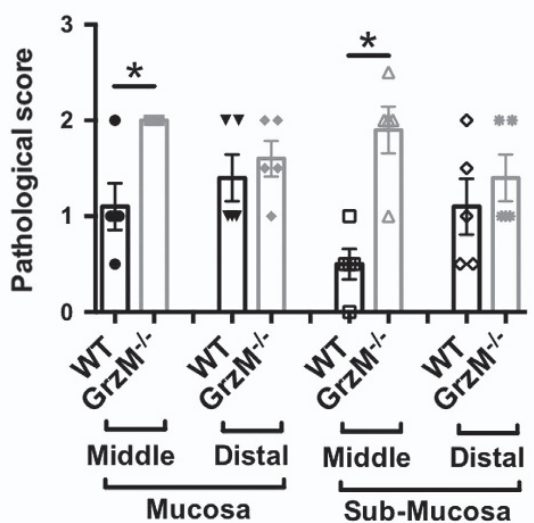

Figure 3 Enhanced inflammation-induced CRC is observed in GrzM-deficient mice. Mice were challenged with AOM/DSS, and samples were assessed according to the experimental design proposed in (a). (b) DSS-induced weight loss was measured twice/week throughout the experimental period of 77 days. Statistical analysis was performed using multiple $T$ tests using the Sidak-Bonferroni method. Results are expressed in mean \pm S.D. $n=15$ mice per group, and ${ }^{*} P<0.05$ was considered for statistical significance. (c) Macroscopical representative images are displayed for one representative WTand one GrzM-deficient mice at the end of the experiment. A cotton stick containing Alcian Blue solution (1\%) was used to swap along the opened colon tissue to enhance the CRC polyp visualization, indicated by the black arrows. (d) Colorectal (CRC) polyp numbers were counted at the end point of the experiment. Statistical analysis was performed using Mann-Whitney test. Results are expressed in mean \pm S.E.M., $n=10$ per group, and ${ }^{* \star} P<0.01$ was considered for statistical significance. (e) Colon length at the end point of the experiment is represented in $\mathrm{cm}$. Statistical analysis was performed using MannWhitney test. Results are expressed in mean \pm S.E.M., $n=5-10$ mice per group, and ${ }^{*} P<0.05$ was considered for statistical significance. (f) Colon histopathology at the end point of the experiment is represented in pathological score from the mucosa and submucosa tissue sections. Statistical analysis was performed using Mann-Whitney test. Results are expressed in mean \pm S.E.M., $n=5$ mice per group, and ${ }^{*} P<0.05$ was considered for statistical significance

(Supplementary Figure 3). Among immune cells, NK cells have been described as the major expressers of GrzM, while expression in other innate T cells (CD8, and $\gamma \delta \mathrm{T})$ can occur at a reduced level. ${ }^{26,34}$ To test whether any of these cells could demonstrate an increased mean fluorescence intensity when labeled with the aa31-257 antibody, intracellular staining and flow-cytometry analysis were performed. However, the aa31-257 staining was again revealed as highly non-specific since all $\mathrm{GrzM}^{-/-}$cells stained positive (Supplementary Figure 4). Given that the mouse anti-human GrzM mAb does not crossreact with mouse $\mathrm{GrzM},{ }^{35}$ these results suggest that there are still no available tools to adequately detect mouse GrzM. 
Role of GrzM in innate immune protection in UC

F Souza-Fonseca-Guimaraes et al

GrzM deficiency results in a distinct cytokine/chemokine profile in the colon during experimental colitis. Several cytokines and chemokines are involved in the 'physiological inflammation' of the normal intestine, and may also have important roles in the pathogenesis of IBD orchestrated by the dynamics of the inflammatory response. ${ }^{30}$ We performed a kinetic analysis to determine which cytokines/chemokines, from a total of 23 targets, would be affected during DSS-induced colitis progression in the distal colonic segment of GrzM-deficient mice (Experimental summary in Table 2). Strikingly, G-CSF, IL-1a, IL-1 $\beta$, IL-17A, MIP1 $a$, MIP1 $\beta$, RANTES, and TNF $a$ levels were elevated in GrzM-deficient mice during disease progression, especially at the end point of the experiment (after 7 days of DSS treatment) (Supplementary Figure 5). In contrast, IFN- $\gamma$, IP-10, and IL-23 were decreased in GrzM-deficient mice during the colitis progression. (Supplementary Figure 6). We failed to detect any GM-CSF, IL-2, and IL-13 cytokines in the colon homogenates (data not shown), while KC, MCP-1, IL-6, IL-10, IL-12p70, IL-21, IL-22, IL-28A/B, and TGF- $\beta 1$ levels were unaffected (data not shown). Our results indicate that the presence of GrzM is critical for the appropriate cytokine/ chemokine response during DSS-induced colitis.

\section{Discussion}

Our current study demonstrates a relationship between GrzM and gut mucosal protection in UC. Grzs are serine proteases produced by cytotoxic lymphocytes that are well known for their potential to induce cell death in target eukaryotic cells. However, previous evidence has shown that GrzM may also participate in inflammatory processes, such as MIP-1a regulation during Listeria monocytogenes infection, and the regulation of secretion of pro-inflammatory cytokines during endotoxic shock. ${ }^{14,17}$ Here, we have shown that GrzM also has a role during the disease progression of UC. Following up on our clinical evidence that GrzM expression is elevated exclusively in the inflamed rectum (distal part of the colon) of UC patients, we have taken advantage of the GrzM-deficient mice to demonstrate in a series of in vivo experiments that host GrzM expression significantly affects the regulation of colonic inflammation. To initially address whether host GrzM would provide a protective advantage or a disadvantage during experimental colitis, we elected the DSS-induced colitis model as a UC experimental model as it has a number of similarities with human UC. ${ }^{19}$ We observed that host GrzM was critical for the protection against colitis, and that its absence results in a significant worsening of colitis in mice following DSS treatment. Surprisingly, the most affected colonic areas in the GrzM-deficient mice were the distal-central segments, which correspond to the distal colon and rectum in UC patients, where we observed elevated GrzM expression during active colitis. These results strongly suggest that GrzM can act directly to induce colonic protection during UC in this specific anatomical region of the gut.

Certain host mutations were described as enhancers of gut permeability, resulting in a dysfunctional immune response to host microbiota, infection by specific pathogens, and/or a defective mucosal barrier to luminal pathogens. ${ }^{1}$ To assess whether GrzM-deficient mice naturally display gut permeability
Table 2 Temporal cytokine/chemokine profiles in distal colon of GrzM-deficient mice during experimental DSS-induced UC

\begin{tabular}{llllll}
\hline & Do & D1 & D3 & D5 & D7 \\
\hline GM-CSF & nd & nd & nd & nd & nd \\
G-CSF & nd & nd & nd & ns & $\uparrow \uparrow \uparrow$ \\
IFN- - & ns & $\downarrow \downarrow \downarrow$ & ns & ns & ns \\
IL-1a & nd & nd & nd & ns & $\uparrow \uparrow \uparrow$ \\
IL-1 $\beta$ & nd & nd & nd & ns & $\uparrow \uparrow \uparrow$ \\
IL-2 & nd & nd & nd & nd & nd \\
IL-6 & nd & nd & ns & ns & ns \\
IL-10 & nd & nd & nd & nd & ns \\
IL-12p70 & ns & ns & ns & ns & ns \\
IL-13 & nd & nd & nd & nd & nd \\
IL-17A & nd & nd & nd & $\uparrow$ & nd \\
IL-21 & ns & ns & ns & ns & ns \\
IL-22 & ns & ns & ns & ns & ns \\
IL-23 & ns & ns & $\downarrow \downarrow$ & ns & ns \\
IL-28A/B & ns & ns & ns & ns & ns \\
IP-10 & ns & $\downarrow$ & ns & ns & ns \\
KC & nd & nd & nd & ns & ns \\
MCP-1 & nd & nd & nd & nd & ns \\
MIP-1a & nd & nd & nd & nd & $\uparrow \uparrow \uparrow$ \\
MIP-1 $\beta$ & nd & nd & nd & ns & $\uparrow \uparrow \uparrow$ \\
RANTES & ns & ns & ns & $\uparrow$ & ns \\
TGF- $\beta 1$ & ns & ns & ns & ns & ns \\
TNFa & nd & nd & nd & $\uparrow$ & ns \\
& & & & & \\
\hline
\end{tabular}

Abbreviations: nd, not detected; ns, not significant. Distal part of colons of WT or GrzM-deficient mice was harvested in different time points after 5\% DSS challenge (days $0,1,3,5$, and 7 ), homogenized in tissue protein extraction buffer, and analyzed for the indicated cytokines/chemokines. Pool of two independent experiments of $n=5$ mice each (total $n=10$ ). Statistical analysis was performed using one-way ANOVA followed by Tukey's post hoc test, where ${ }^{\star} P<0.05$ ( $\uparrow$ for significantly higher, or $\downarrow$ for significantly lower than WT), ${ }^{\star \star} P<0.01$ ( $\uparrow \uparrow$ for significantly higher, or $\downarrow \downarrow$ for significantly lower than WT), and ${ }^{\star * \star} P<0.001$ ( $\uparrow \uparrow \uparrow$ for significantly higher, or $\downarrow \downarrow \downarrow$ for significantly lower than WT) were considered for statistical significance.

- Pro-inflammatory neutrophil-related cytokines/chemokines analyzed: GM-CSF, G-CSF, IL-17A, and KC.

- Chemokines analyzed: IP-10, MCP-1, MIP-1 $\alpha$, MIP-1 $\beta$, and RANTES

- Pro-inflammatory cytokines analyzed: IFN- $\gamma$, IL-1 $\alpha$, IL-1 $\beta$, IL-2, IL-6, IL-12, IL-13, IL-21, IL-23, IL-28A/B, and TNFa.

- Anti-inflammatory, tissue repair proteins: IL-10, IL-22, and TGF- $\beta 1$.

at steady state, or whether it is enhanced during DSS challenge, we performed a gut integrity assay based on FITC-dextran leakage from the gut to the blood compartment. We have identified that gut integrity was not compromised at steady state, but worsened during DSS treatment. Our results also reinforced the finding that enhanced colon damage in GrzM-deficient mice seemed to be limited to the experimental model used, as no such phenotype was observed in other gutrelated infection/pathology models including Toxoplasma gondii and Citrobacter rodentium infections. During inflammatory tissue damage occurring in UC, inflamed tissues typically represent trans-epithelial migration of neutrophils, which can alter the gut barrier function by enhancing the epithelial paracellular permeability during disease progression. ${ }^{36}$ Along with the enhanced pathology and increased gut permeability seen during DSS treatment, as expected, we also observed an enhanced neutrophil infiltration in the colonic lamina propria in GrzM-deficient mice.

IBD-induced CRC is a subsequent clinical complication that can account for up to $15 \%$ of all deaths among IBD patients, and IBD patients are at least six times more likely to develop CRC. To explore whether host GrzM is involved in protection against CRC development, we assessed well-established 
models that mimic both IBD-induced CRC in GrzM-deficient mice and their respective WT controls. ${ }^{32}$ As expected, the absence of GrzM expression resulted in an enhanced AOM-DSS-induced CRC, which is exclusively dependent on enhanced colonic inflammation. Another study recently showed that GrzM expression in epithelial cells contributes to chemoresistance and epithelial-mesenchymal transition (EMT) during colon carcinogenesis, demonstrating that GrzM expression increases in colon cancer tissues and cell lines upon enhanced EMT phenotype. ${ }^{33}$ We could not assess EMT in our model, but when we tested the anti-mouse GrzM antibodies used in the study by Wang et al., ${ }^{33}$ these reagents were highly non-specific as determined by negative control GrzM-deficient samples. Precise cellular GrzM expression assays remain controversial and limited, as there is still no efficient/specific method to detect GrzM in mice, and GrzMreporter mice are still not available. A technique of tagging GrzM with a fluorescent label and then using live-cell imaging may demonstrate whether GrzM is secreted from NK cells or whether it has intracellular functions instead; however, a limiting factor is that recombinant GrzM is not commercially available.

Several cytokines and chemokines have an important role in the pathogenesis of IBD, and modulation of specific cytokines is a current clinical therapeutic strategy (e.g., neutralizing antibodies to TNFa). ${ }^{30}$ Considering that different colonic segments can display different colitis intensities according to our previous data, we homogenized in tissue protein extraction buffers and screened 23 cytokines/chemokines from the distal colon segments at different time points following DSS treatment. Certain pro-cytokines and chemokines were elevated at later time points following DSS treatment in the mice: G-CSF, IL-1a, IL-1 $\beta$, IL-6, IL-17A, MIP-1a, MIP-1 $\beta$, RANTES, and TNFa. IL-1 cytokines and TNF $a$ are classically produced by macrophages or dendritic cells (DCs) via TLR sensing of the commensal microbiota. ${ }^{37}$ TNFa neutralization, but not IL-1 neutralization, was already shown to be effective in DSS-induced colitis by favoring mucosal healing. ${ }^{38}$ In addition, IL-1 and TNFa were also demonstrated to be associated with colorectal tumorigenesis by contributing to tissue inflammation-induced oncogenesis. ${ }^{39,40}$ This evidence corroborates our findings of enhanced colitis and inflammationinduced tumorigenesis outcomes in GrzM-deficient mice subjected to DSS challenge. Chemokines are also critical factors in regulating immune cell trafficking to inflammatory sites. MIP-1a, MIP-1 $\beta$, and RANTES are chemokines that have already previously been shown to contribute to the exacerbation of IBD in experimental mouse models via overzealous neutrophil attraction and infiltration of the colonic tissues. ${ }^{41-44}$ In addition, G-CSF and IL-17A are important cytokines that activate neutrophil innate functions to control bacterial infection, but in excess, they have already been described as contributing to exaggerated neutrophil activation and consequent tissue damage during DSSinduced colitis. ${ }^{45,46}$ This evidence can provide an explanation for our observations of enhanced neutrophil infiltration of the colonic tissues of GrzM-deficient mice after DSS treatment, and the associated tissue damage and enhanced disease outcome. IFN- $\gamma$ and IFN- $\gamma$-induced protein (IP-10) were also observed in lower concentrations in the GrzM-deficient

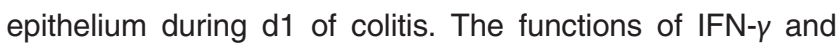
IP-10 are to promote Th1 differentiation and to attract these cells to the inflammation site, respectively. ${ }^{47,48}$ Th1-related cells and cytokines were previously shown to represent a favorable prognostic sign in human CRC initiation. ${ }^{49}$ The decreased IFN- $\gamma$ and IP-10 levels in distal colon homogenates might also suggest that the anticancer Th1 response may be reduced in GrzM-deficient mice. Further research is necessary to elucidate the specific stage of the disease progression in which GrzM has a role, and to demonstrate that this enzyme might offer complementary benefits for treating or preventing UC.

\section{Materials and Methods}

Clinical samples, RNA isolation, and sequencing. Patients with a confirmed diagnosis of either UC or CD undergoing colonoscopy for surveillance or assessment of their disease, and healthy controls undergoing screening colonoscopy because of a family history of CRC, were recruited into an established, ethically-approved study investigating the natural history and pathogenesis of IBD.

Mice. C57BL/6J WT mice were purchased from the Walter and Eliza Hall Institute of Medical Research and housed at the QIMR Berghofer Medical Research Institute. C57BL/6 GrzM-deficient mice were previously described by our group, ${ }^{18}$ and were bred at the QIMR Berghofer Medical Research Institute. C57BL/6 perforin (Prf1)-deficient ${ }^{50}$ and TCR $\delta$-deficient mice ${ }^{51}$ were bred at the QIMR Berghofer Medical Research Institute. Conditional transgenic NK cell-deficient mice (NKp46cre $\times \mathrm{Mcl}^{\mathrm{ft}} \mathrm{f}^{\mathrm{flfl}}$ ) were previously described by our group, ${ }^{52}$ and were bred at the Walter and Eliza Hall Institute of Medical Research. In certain experiments, anti-CD8 (clone 53.5.8), or respectively control lgG (control group), was administered in a $100 \mu \mathrm{g}$ i.p. dose at day -1 , day 0 , and day 7 relatively to DSS administration to induce CD8 T-cell depletion. ${ }^{53}$ All mice used were females between the ages of 8 and 14 weeks. All experiments were approved by QIMR Berghofer Medical Research Institute and Walter and Eliza Hall Institute of Medical Research animal ethics committees.

In vivo DSS-induced IBD. To induce colitis, WT and other indicated genotypes/antibody-depletion group were treated with either 0 or $5 \%$ DSS (molecular mass 40-50 kDa; USB, Affymetrix Inc, Ohio, USA), dissolved in their sterile drinking water and provided ad libitum over a 4-day period to simulate the inflammatory conditions experienced during IBD, according to a previously described protocol. ${ }^{29} \mathrm{~A}$ baseline weight was recorded for each mouse before treatment and weight was then monitored daily for the subsequent 2-week period. After 4 days, the DSS treatment was replaced with normal autoclaved water to allow for inflammation progression and resolution. On days 7 (acute inflammation) and 14 (tissue recovery period) post DSS treatment, mice were killed via $\mathrm{CO}_{2}$ asphyxiation, and biopsies were taken to investigate the inflammatory conditions.

In vivo AOM-DSS-induced CRC. AOM/DSS-induced CRC was performed as previously described. ${ }^{32}$ Briefly, WT and GrzM-deficient mice were individually tagged, had their body weights measured, and were injected i.p. with $10 \mathrm{mg} / \mathrm{kg}$ of AOM (Sigma-Aldrich, St. Louis, MO, USA) diluted in PBS. After 7 days post AOM treatment, $2.5 \%$ DSS was administered in their drinking water to simulate the chronic inflammatory conditions experienced prior the development of CRC polyps. After 7 days, the DSS treatment was replaced by normal autoclaved water to allow for inflammation progression and resolution during the following 2 weeks. Another two cycles of $2.5 \%$ DSS treatment for 7 days and normal drinking water for 14 days were performed. During the treatment period, weight was monitored twice a week. After 77 days post AOM injection (tissue recovery period), mice were killed via $\mathrm{CO}_{2}$ asphyxiation, and biopsies were taken to investigate the inflammatory conditions.

Histology and histopathology assessment. Colonic tissues from unchallenged, DSS, or AOM-DSS treated mice were rolled into Swiss rolls and fixed in PBS-Formalin 10\% for $48 \mathrm{~h}$ and then submitted for paraffin processing and HE or GRAM staining performed routinely by the QIMR Berghofer Medical Institute's Histology Facility. Slides were then scanned using an Aperio XT slide scanner using $\times 40$ scan magnification. Histopathology in colon samples from DSS-induced UC experiments was assessed as previously described, ${ }^{20}$ using the following 
parameters for histopathological scoring: Crypt integrity: $0=$ normal; $1=$ irregular crypts, including the presence of apoptosis; $2=$ mild crypt loss, and/or mild epithelial loss from surface of bowel; $3=$ severe crypt loss; $4=$ complete crypt loss with an intact epithelial cell layer; $5=$ complete loss of crypts and surface epithelium (<crypt width); and $6=$ complete loss of crypts and surface epithelium (>crypt width). Infiltration of muscle: $0=$ normal; $1=$ mild; $2=$ moderate; and $3=$ severe. Infiltration of inflammatory cells into mucosa: $0=$ normal; $1=$ mild; $2=$ moderate; and $3=$ severe. Infiltration of inflammatory cells into submucosa: $0=$ normal; $1=$ mild; $2=$ moderate; and $3=$ severe. Scores were added, resulting in a total scoring range of 1-17. Histopathology in colon samples from AOM-DSS-induced CRC experiments was assessed as previously described by our group ${ }^{54}$ using the following parameters for histopathological scoring: Epithelial integrity: $0=$ normal; $1=$ hyper proliferation; $2=<50 \%$ crypt loss; $3=>50 \%$ crypt loss; $4=$ complete crypt loss; and $5=$ ulcer. Presence of inflammatory cells: $0=$ none; $1=$ mild; $2=$ moderate; and $3=$ severe. Scores were added, resulting in a total scoring range of $0-8$.

Sample preparation for flow-cytometry analysis. Mesenteric lymph nodes and spleens were homogenized in PBS into $40 \mu \mathrm{m}$ cell strainers, followed by red blood cell (RBC) lysis. Colonic lamina propria and intraepithelial leukocytes (IELs) were prepared as previously described. ${ }^{22}$ Fresh colons were harvested from $\mathrm{CO}_{2}$ killed mice, cleaned by flushing $10 \mathrm{ml}$ of cold PBS using a gavage syringe needle, cut into $\sim 0.5 \mathrm{~cm}$ sections and dissociated in $2 \% \mathrm{FCS} \mathrm{HANKS} \mathrm{Ca}^{+} \mathrm{Mg}^{+}$-free media with $5 \mathrm{mM}$ EDTA for $30 \mathrm{~min}$ at $37^{\circ} \mathrm{C}$ with gentle rotation to obtain the IEL fraction (supernatant). The subsequent tissues were then digested for $45 \min$ in $2 \%$ FCS RPMl $2 \mathrm{mg} / \mathrm{ml}$ Collagenase III (Worthington Biochemical, Lakewood, NJ, USA), 0.4 U Dispase (Invitrogen, Thermo Fisher Scientific, Grand Island, NY, USA), and DNAse $1 \mu \mathrm{g} / \mathrm{ml}$ (Roche Diagnostics USA, Indianapolis, IN, USA) for isolation of lamina propria leukocytes. Cells from both IEL and lamina propria fractions were further purified by centrifugation on $40 \% / 80 \%$ Percoll gradient for 20 min at $900 \times g$ without breaks. Cells harvested from single-cell suspensions from various organs were incubated for $15 \mathrm{~min}$ in Fc blocking buffer (2.4G2 antibody). Cells were then stained with the following antibodies: anti-mouse -B220 (RA3-6B2), -CD3 (17A2), -CD4 (GK1.5), -CD8 (53-6.7) -CD19 (1D3), -Foxp3 (FJK-16S) - $\gamma \delta$ TCR (GL3), -Ly6C (HK1.4), -Ly6G (1A8), -NKp46 (29A1.4), -NK1.1 (PK136), and -TCR (H57-597). All mAbs were purchased from eBiosciences (San Diego, CA, USA), BD Biosciences (San Jose, CA, USA), or Biolegend (San Diego, CA, USA). Zombie Yellow, or Zombie UV, Fixable Viability Kit (Biolegend) was used to assess viability. Acquisition was performed using LSR II Fortessa Flow Cytometer (BD Biosciences). Analysis was achieved using Flowjo (Tree Star, Ashland, OR, USA) software.

Colon homogenates and cytokine detection. Fresh colons biopsies from unchallenged (d0) or 5\% DSS-treated mice (d1, d3, d5, and d7) were flushed with $10 \mathrm{ml}$ of cold PBS using a gavage syringe needle for stool content cleaning. Colons were then gently dried with absorbent tissue, length was measured with support of a ruler to allow the separation of distal colon parts, which were then weighed and homogenized as previously described ${ }^{55}$ using T-PER Tissue Protein Extraction Reagent (Thermo Fisher Scientific Life Sciences, Waltham, MA, USA; supplemented with PhosSTOP Phosphatase and complete Mini protease inhibitor tablets (Roche)), according to the manufacturer's instructions. All samples were stored at $-80^{\circ} \mathrm{C}$ until analysis. After filtering samples in $40 \mu \mathrm{m}$ filters, and centrifugation at $10000 \times g$ to pellet debris, the concentrations of a panel of cytokines and chemokines in supernatants were measured as previously described. ${ }^{56}$ The detection methods applied were either by using Cytometric Bead Array (CBA) technology (BD Biosciences) according to the manufacturer's instructions (for GM-CSF, G-CSF, IL-1 $\alpha$, IL-1 $\beta$, IL-2, IL-6, IL-10, IL-13, IL-17A, KC, MCP-1, MIP- $1 \alpha$, MIP- $1 \beta$, RANTES, TNF $\alpha$ ) or by using ELISA Duoset Kits (R\&D Systems, Minneapolis, MN, USA) according to the manufacturer's instructions (for IFN- $\gamma$, IL-12p70, IL-21, IL-22, IL-23, IL-28A/B, TGF- $\beta 1$ ). In all cases, cytokine levels were normalized to the weight of the respective colon explant.

Gut integrity assay. Gut integrity was assessed before or after 4 days of treatment with $5 \%$ DSS as previously described. ${ }^{25}$ Naive or DSS-treated mice (d7 post DSS) were kept without food or water for $4 \mathrm{~h}$, and treated by oral gavage with $300 \mu \mathrm{l}$ of FITC-dextran molecular weight 4000 (Sigma-Aldrich) at $60 \mathrm{mg} / \mathrm{ml}$. Plasma was obtained after $4 \mathrm{~h}$ of FITC-dextran treatment, diluted $1: 1$ with PBS and fluorescence was read in a plate reader $(485 / 535 \mathrm{~nm})$ using a standard curve.

Statistical analysis. Statistical analysis was achieved using Graph Pad Prism (La Jolla, CA, USA) or SPSS Software (Chicago, IL, USA). Data were considered to be statistically significant where the $P$-value was equal to or less than 0.05 . Statistical tests used were the Mann-Whitney test or one-way ANOVA with Tukey's post hoc test for treatments/genotypes comparison, multiple $T$ tests using the Sidak-Bonferroni method for weight loss comparison, and the Mantel-Cox Log Rank test for survival.

\section{Conflict of Interest}

The authors declare no conflict of interest.

Acknowledgements. We thank Clay Winterford, Nigel Waterhouse, Lisa Simms, Lutz Krause, Martha Zakrzewski, Motti Gerlic, Simon Keely, Stephen A Wilcox, and Tobias Bald for providing reagents, discussion, comments, and advice in this project, and Cathy Quilici, Eren Loza, Kate Elder, Leesa Wockner, Louise Spencer, Rebecca Delconte, and Tam Nguyen for technical support. MJS was supported by an NHMRC Senior Principal Research Fellowship. FSFG was supported by an NHMRC Early Career Fellowship and National Breast Cancer Foundation (NBCF) Fellowship, and Cancer Cure Australia Priority-driven Young Investigator Project Grant.

\section{Author contributions}

FS-F-G, GR-S, LT, and MJS designed research, and supervised work. FS-F-G, MJS, and GR-S wrote the paper. FS-F-G, GTB, KM, KPM, HT, LAM, LM, NDH, SLM, WS, and YK performed research. FS-F-G, MZ, GCG, TP, MJS, and GRS analyzed data.

1. Hanauer SB. Inflammatory bowel disease: epidemiology, pathogenesis, and therapeutic opportunities. Inflamm Bowel Dis 2006; 12(Suppl 1): S3-S9.

2. Graham DB, Xavier RJ. From genetics of inflammatory bowel disease towards mechanistic insights. Trends Immunol 2013; 34: 371-378.

3. Cosnes J, Gower-Rousseau C, Seksik P, Cortot A. Epidemiology and natural history of inflammatory bowel diseases. Gastroenterology 2011; 140: 1785-1794.

4. Kim ER, Chang DK. Colorectal cancer in inflammatory bowel disease: the risk, pathogenesis, prevention and diagnosis. World J Gastroenterol 2014; 20: 9872-9881.

5. Marchal Bressenot A, Riddell RH, Boulagnon-Rombi C, Reinisch W, Danese S, Schreiber S et al. Review article: the histological assessment of disease activity in ulcerative colitis. Aliment Pharmacol Ther 2015; 42: 957-967.

6. Trapani JA, Smyth MJ. Functional significance of the perforin/granzyme cell death pathway. Nat Rev Immunol 2002; 2: 735-747.

7. Voskoboinik I, Smyth MJ, Trapani JA. Perforin-mediated target-cell death and immune homeostasis. Nat Rev Immunol 2006; 6: 940-952.

8. Wensink AC, Hack CE, Bovenschen N. Granzymes regulate proinflammatory cytokine responses. J Immunol 2015; 194: 491-497.

9. Anthony DA, Andrews DM, Watt SV, Trapani JA, Smyth MJ. Functional dissection of the granzyme family: cell death and inflammation. Immunol Rev 2010; 235: 73-92.

10. Walch M, Dotiwala F, Mulik S, Thiery J, Kirchhausen T, Clayberger $C$ et al. Cytotoxic cells kill intracellular bacteria through granulysin-mediated delivery of granzymes. Cell 2014; 157: 1309-1323.

11. Tew GW, Hackney JA, Gibbons D, Lamb CA, Luca D, Egen JG et al. Association between response to etrolizumab and expression of integrin alpha $E$ and granzyme $A$ in colon biopsies of patients with ulcerative colitis. Gastroenterology 2016; 150: 477-487.

12. Smyth MJ, Wiltrout T, Trapani JA, Ottaway KS, Sowder R, Henderson LE et al. Purification and cloning of a novel serine protease, RNK-Met-1, from the granules of a rat natural killer cell leukemia. J Biol Chem 1992; 267: 24418-24425.

13. Sayers TJ, Brooks AD, Ward JM, Hoshino T, Bere WE, Wiegand GW et al. The restricted expression of granzyme M in human lymphocytes. J Immunol 2001; 166: 765-771.

14. Anthony DA, Andrews DM, Chow M, Watt SV, House C, Akira S et al. A role for granzyme M in TLR4-driven inflammation and endotoxicosis. J Immunol 2010; 185: 1794-1803.

15. Smyth MJ, O'Connor MD, Trapani JA, Kershaw MH, Brinkworth RI. A novel substrate-binding pocket interaction restricts the specificity of the human NK cell-specific serine protease, Metase-1. J Immunol 1996; 156: 4174-4181.

16. Mahrus S, Kisiel W, Craik CS. Granzyme $M$ is a regulatory protease that inactivates proteinase inhibitor 9, an endogenous inhibitor of granzyme B. J Biol Chem 2004; 279: 54275-54282.

17. Baschuk N, Wang N, Watt SV, Halse H, House C, Bird PI et al. NK cell intrinsic regulation of MIP-1alpha by granzyme M. Cell Death Dis 2014; 5: e1115.

18. Pao LI, Sumaria N, Kelly JM, van Dommelen S, Cretney E, Wallace ME et al. Functional analysis of granzyme $M$ and its role in immunity to infection. $J$ Immunol 2005; 175 3235-3243.

19. Chassaing B, Aitken JD, Malleshappa M, Vijay-Kumar M. Dextran sulfate sodium (DSS)-induced colitis in mice. Curr Protoc Immunol 2014; 104: Unit 1525. 
20. Kirkland D, Benson A, Mirpuri J, Pifer R, Hou B, DeFranco AL et al. B cell-intrinsic MyD88 signaling prevents the lethal dissemination of commensal bacteria during colonic damage. Immunity 2012; 36: 228-238.

21. Gorfu G, Cirelli KM, Melo MB, Mayer-Barber K, Crown D, Koller BH et al. Dual role for inflammasome sensors NLRP1 and NLRP3 in murine resistance to Toxoplasma gondii. $\mathrm{mBio}$ 2014 8; 5(1). pii: e01117-13.

22. Rankin LC, Groom JR, Chopin M, Herold MJ, Walker JA, Mielke LA et al. The transcription factor T-bet is essential for the development of NKp46+ innate lymphocytes via the Notch pathway. Nat Immunol 2013; 14: 389-395.

23. Fournier BM, Parkos CA. The role of neutrophils during intestinal inflammation. Mucosal Immunol 2012; 5: 354-366.

24. Laukoetter MG, Nava P, Lee WY, Severson EA, Capaldo CT, Babbin BA et al. JAM-A regulates permeability and inflammation in the intestine in vivo. J Exp Med 2007; 204: 3067-3076.

25. Brandl K, Rutschmann S, Li X, Du X, Xiao N, Schnabl B et al. Enhanced sensitivity to DSS colitis caused by a hypomorphic Mbtps1 mutation disrupting the ATF6-driven unfolded protein response. Proc Natl Acad Sci USA 2009; 106: 3300-3305.

26. Krenacs L, Smyth MJ, Bagdi E, Krenacs T, Kopper L, Rudiger T et al. The serine protease granzyme $M$ is preferentially expressed in NK-cell, gamma delta T-cell, and intestinal T-cell lymphomas: evidence of origin from lymphocytes involved in innate immunity. Blood 2003; 101: 3590-3593.

27. Seillet C, Belz GT, Huntington ND. Development, Homeostasis, and Heterogeneity of NK Cells and ILC1. Curr Top Microbiol Immunol 2016; 395: 37-61.

28. Lopez JA, Susanto O, Jenkins MR, Lukoyanova N, Sutton VR, Law RH et al. Perforin forms transient pores on the target cell plasma membrane to facilitate rapid access of granzymes during killer cell attack. Blood 2013; 121: 2659-2668.

29. Whittem CG, Williams AD, Williams CS. Murine colitis modeling using dextran sulfate sodium (DSS). J Vis Exp 2010; 19: pii 1652.

30. Neurath MF. Cytokines in inflammatory bowel disease. Nat Rev Immunol 2014; 14: 329-342.

31. Terzic J, Grivennikov S, Karin E, Karin M. Inflammation and colon cancer. Gastroenterology 2010; 138: 2101-2114 e2105.

32. Putoczki TL, Thiem S, Loving A, Busuttil RA, Wilson NJ, Ziegler PK et al. Interleukin-11 is the dominant IL- 6 family cytokine during gastrointestinal tumorigenesis and can be targeted therapeutically. Cancer Cell 2013; 24: 257-271.

33. Wang H, Sun Q, Wu Y, Wang L, Zhou C, Ma W et al. Granzyme M expressed by tumor cells promotes chemoresistance and EMT in vitro and metastasis in vivo associated with STAT3 activation. Oncotarget 2015; 6: 5818-5831.

34. Smyth MJ, Browne KA, Thia KY, Apostolidis VA, Kershaw MH, Trapani JA. Hypothesis: cytotoxic lymphocyte granule serine proteases activate target cell endonucleases to trigger apoptosis. Clin Exp Pharmacol Physiol 1994; 21: 67-70.

35. Smyth MJ, O'Connor MD, Kelly JM, Ganesvaran P, Thia KY, Trapani JA. Expression of recombinant human Met-ase-1: a NK cell-specific granzyme. Biochem Biophys Res Commun 1995; 217: 675-683.

36. Kucharzik T, Walsh SV, Chen J, Parkos CA, Nusrat A. Neutrophil transmigration in inflammatory bowel disease is associated with differential expression of epithelial intercellular junction proteins. Am J Pathol 2001; 159: 2001-2009.

37. Ng SC, Benjamin JL, McCarthy NE, Hedin CR, Koutsoumpas A, Plamondon S et al. Relationship between human intestinal dendritic cells, gut microbiota, and disease activity in Crohn's disease. Inflamm Bowel Dis 2011; 17: 2027-2037.

38. Kojouharoff G, Hans W, Obermeier F, Mannel DN, Andus T, Scholmerich $\mathrm{J}$ et al Neutralization of tumour necrosis factor (TNF) but not of IL-1 reduces inflammation in chronic dextran sulphate sodium-induced colitis in mice. Clin Exp Immunol 1997; 107: 353-358.

39. Popivanova BK, Kitamura K, Wu Y, Kondo T, Kagaya T, Kaneko S et al. Blocking TNF-alpha in mice reduces colorectal carcinogenesis associated with chronic colitis. J Clin Invest 2008; 118: $560-570$

40. Wang Y, Wang K, Han GC, Wang RX, Xiao H, Hou CM et al. Neutrophil infiltration favors colitis-associated tumorigenesis by activating the interleukin-1 (IL-1)/IL-6 axis. Mucosal Immunol 2014; 7: 1106-1115.

41. Pender SL, Chance V, Whiting CV, Buckley M, Edwards M, Pettipher R et al. Systemic administration of the chemokine macrophage inflammatory protein 1alpha exacerbates inflammatory bowel disease in a mouse model. Gut 2005; 54: 1114-1120.
42. Ajuebor MN, Hogaboam CM, Kunkel SL, Proudfoot AE, Wallace JL. The chemokine RANTES is a crucial mediator of the progression from acute to chronic colitis in the rat. $J$ Immunol 2001; 166: 552-558.

43. Ajuebor MN, Swain MG. Role of chemokines and chemokine receptors in the gastrointestinal tract. Immunology 2002; 105: 137-143

44. Shea-Donohue T, Thomas K, Cody MJ, Aiping Z, Detolla LJ, Kopydlowski KM et al. Mice deficient in the CXCR2 ligand, CXCL1 (KC/GRO-alpha), exhibit increased susceptibility to dextran sodium sulfate (DSS)-induced colitis. Innate Immun 2008; 14: 117-124.

45. Ito R, Kita M, Shin-Ya M, Kishida T, Urano A, Takada R et al. Involvement of IL-17A in the pathogenesis of DSS-induced colitis in mice. Biochem Biophys Res Commun 2008; 377 : 12-16.

46. Ina K, Kusugami K, Hosokawa T, Imada A, Shimizu T, Yamaguchi T et al. Increased mucosal production of granulocyte colony-stimulating factor is related to a delay in neutrophil apoptosis in inflammatory bowel disease. J Gastroenterol Hepatol 1999; 14: 46-53.

47. Taub DD, Lloyd AR, Conlon K, Wang JM, Ortaldo JR, Harada et al. Recombinant human interferon-inducible protein 10 is a chemoattractant for human monocytes and $T$ lymphocytes and promotes T cell adhesion to endothelial cells. J Exp Med 1993; 177 : 1809-1814.

48. Martin-Fontecha A, Thomsen LL, Brett S, Gerard C, Lipp M, Lanzavecchia et al. Induced recruitment of $\mathrm{NK}$ cells to lymph nodes provides IFN-gamma for $\mathrm{T}(\mathrm{H}) 1$ priming. Nat Immunol 2004; 5: 1260-1265

49. Pages F, Berger A, Camus M, Sanchez-Cabo F, Costes A, Molidor R et al. Effector memory T cells, early metastasis, and survival in colorectal cancer. N Engl J Med 2005; 353: 2654-2666.

50. Kagi D, Ledermann B, Burki K, Seiler P, Odermatt B, Olsen KJ et al. Cytotoxicity mediated by T cells and natural killer cells is greatly impaired in perforin-deficient mice. Nature 1994; 369 : 31-37.

51. Fujihashi K, McGhee JR, Kweon MN, Cooper MD, Tonegawa S, Takahashi I et al. gamma/ delta $T$ cell-deficient mice have impaired mucosal immunoglobulin A responses. J Exp Med 1996; 183: 1929-1935.

52. Sathe P, Delconte RB, Souza-Fonseca-Guimaraes F, Seillet C, Chopin M, Vandenberg CJ et al. Innate immunodeficiency following genetic ablation of Mcl1 in natural killer cells. Nat Commun 2014; 5: 4539.

53. Ferrari de Andrade L, Ngiow SF, Stannard K, Rusakiewicz S, Kalimutho M, Khanna KK et al. Natural killer cells are essential for the ability of BRAF inhibitors to control BRAFV600Emutant metastatic melanoma. Cancer Res 2014; 74: 7298-7308.

54. Mielke L, Preaudet A, Belz G, Putoczki T. Confocal laser endomicroscopy to monitor the colonic mucosa of mice. J Immunol Methods 2015; 421: 81-88.

55. Ey B, Eyking A, Klepak M, Salzman NH, Gothert JR, Runzi M et al. Loss of TLR2 worsens spontaneous colitis in MDR1A deficiency through commensally induced pyroptosis. $\mathrm{J}$ Immunol 2013; 190: 5676-5688.

56. Souza-Fonseca-Guimaraes F, Young A, Mittal D, Martinet L, Bruedigam C, Takeda K et al. NK cells require IL-28R for optimal in vivo activity. Proc Natl Acad Sci USA 2015; 112: E2376-E2384.

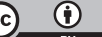

Cell Death and Disease is an open-access journal published by Nature Publishing Group. This work is licensed under a Creative Commons Attribution 4.0 International License. The images or other third party material in this article are included in the article's Creative Commons license, unless indicated otherwise in the credit line; if the material is not included under the Creative Commons license, users will need to obtain permission from the license holder to reproduce the material. To view a copy of this license, visit http://creativecommons.org/licenses/by/4.0/

(C) The Author(s) 2016

Supplementary Information accompanies this paper on Cell Death and Disease website (http://www.nature.com/cddis) 